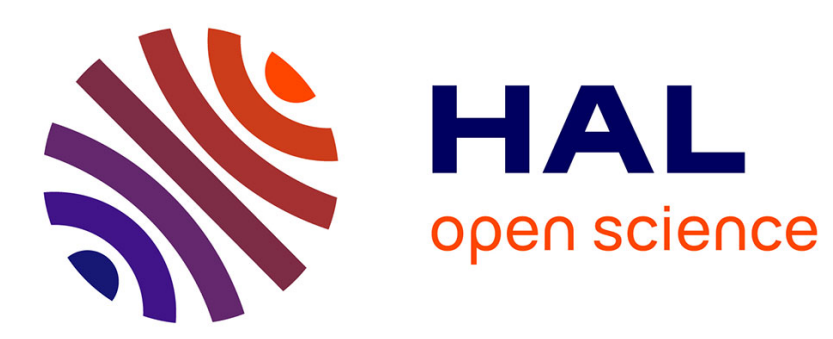

\title{
Interface Between Simple Crystal and Icosahedral-Symmetry Related Crystal
}

A. Proult, A. Redjaïmia, P. Donnadieu

\section{To cite this version:}

A. Proult, A. Redjaïmia, P. Donnadieu. Interface Between Simple Crystal and IcosahedralSymmetry Related Crystal. Journal de Physique IV Proceedings, 1996, 06 (C2), pp.C2-135-C2-140. 10.1051/jp4:1996218 . jpa-00254196

\section{HAL Id: jpa-00254196 https://hal.science/jpa-00254196}

Submitted on 1 Jan 1996

HAL is a multi-disciplinary open access archive for the deposit and dissemination of scientific research documents, whether they are published or not. The documents may come from teaching and research institutions in France or abroad, or from public or private research centers.
L'archive ouverte pluridisciplinaire HAL, est destinée au dépôt et à la diffusion de documents scientifiques de niveau recherche, publiés ou non, émanant des établissements d'enseignement et de recherche français ou étrangers, des laboratoires publics ou privés. 


\title{
Interface Between Simple Crystal and Icosahedral-Symmetry Related Crystal
}

\author{
A. Proult, A. Redjaïmia* and P. Donnadieu \\ Laboratoire d'Etudes des Microstructures, CNRS/ONERA, 29 av. Division Leclerc, BP. 72, \\ 92322 Châtillon sous Bagneux cedex, France \\ * LSGS, URA 1402 du CNRS, EMN-EEIGM, Parc de Saurupt, 54042 Nancy cedex, France
}

\begin{abstract}
The phase diagram of transition metal intermetallics exhibits a systematic sequence of phases involving simple crystals and complex crystal structures with icosahedral motifs ( $B-W, \sigma, \chi$ phases). Such intermetallic alloys provide then convenient examples to establish basis for the analysis of simple crystal/ icosahedral phase interfaces. The $\sigma$ and $\chi$ intermetallic phases in a Fe-Cr-Mo steel have been studied by Transmission Electron Microscopy: the interface as well as the orientation relationships has been identified. A simple geometrical analysis is proposed to account for the ferrite/ $\sigma$-phase interface, $\sigma / \chi$ orientation relationship and $\chi$-phase antiphase boundaries. This approach emphasizes on the necessity to consider corrugated compact layers instead of lattice planes in icosahedral phases and the conservation of the site density at the interface plane.
\end{abstract}

\section{INTRODUCTION}

In intermetallic compounds, phase diagrams have always been of great concern since they allow to understand and predict the stability of the different phases in equilibrium conditions. Nevertheless, most of the metallurgical cases cannot be considered as equilibrium states. For instance, solid state transformations, such as precipitation, never occur at equilibrium. Understanding their mechanism requires to consider the free energy as well as the interface energy.

It is usually stated that the interface energy is reduced by the structural similarity exhibited by both phases at the interface. The orientation relationships (O.R.) between precipitates and matrix are inherited from the interface crystallographic properties. Of course, atomic diffusion, especially by controlling the kinetic, has a key role in precipitation. But the fact that the structural relationships are established in the earlier stage of precipitation and are maintained during many diffusional transformations confirms that interface and O.R. are rather controlled by the structural (= geometrical) relations than by the chemical changes [1].

As far as simple intermetallic crystals ${ }^{1}$ are concerned, the structural similarities are easy to identify and simple features have been observed: the interface is usually a dense atomic plane for each phase (matrix and precipitate). When two phases, very different from the crystallographic point of view, have to be compared this approach cannot be applied because lattice planes are atomic planes only in simple crystals. Of course, interfaces between simple crystal and icosahedral quasicrystal stand as the extreme case but the difficulties already exist for interface between simple crystal and crystalline phases involving non-crystallographic motifs. Such crystals do exist in intermetallic compounds; they are either called Frank-Kasper phases or TCP phases because their atomic decoration obeys to a Topological Close Packing (TCP) rule [2]. Because of the packing rule, the atom coordination is described by an icosahedron or polyhedron related to the icosahedron. Since the atomic decoration involves icosahedral motifs, these phases are also considered as approximant crystals of the icosahedral quasicrystals. In both cases (quasicrystal or Frank-Kasper phases), there is no more compact atomic planes because the topological packing rule leads to corrugated atom layers as demonstrated by Frank and Kasper [2].

In the following, our analysis of interface between simple crystals and icosahedral motif crystals does not rely on the existence of the periodicity. Therefore, for brievity, we will use frequently "icosahedral phase" instead of "crystal with icosahedral motifs". Actually, valuable information for further studies of crystal/quasicrystal interface is expected from the interface between simple crystal and crystals with

\footnotetext{
1 in the present work simple crystal means metallic crystal with few atoms per cell, by opposition to crystals with large cell decorated with icosahedral motifs exemplified here by the $\sigma$ and $\chi$ phases
} 
icosahedral motifs. Besides, the atomic decoration of quasicrystalline phases is still under investigation while the cell decorations in Frank-Kasper phases are well known.

Transition metal intermetallics provide the most interesting case since their phase diagrams exhibit a systematic sequence of simple crystals and complex phases [3]. This sequence is constituted by simple b.c.c. and f.c.c. crystals separated by Frank-Kasper phases which belong to classical structure-types ( $B-W$, $\sigma$ and $\alpha-\mathrm{Mn}$ ). Though one of the complex phases may be absent, the following order is always observed:

$$
\text { b.c.c. } \rightarrow \mathrm{B}-\mathrm{W} \rightarrow \sigma \rightarrow \alpha-\mathrm{Mn} \rightarrow \text { f.c.c. }
$$

An adequate example for studying the microstructure of precipitation is provided by the $\mathrm{Fe}-\mathrm{Cr}-\mathrm{Ni}-\mathrm{Mo}$ system. In duplex steels (wt\% $69 \mathrm{Fe}-23 \mathrm{Cr}-5 \mathrm{Ni}-3 \mathrm{Mo}$ ), precipitation of $\sigma$ and $\chi$ phase particles $(\chi$ is isostructural to $\alpha-\mathrm{Mn}$ ) occurs after annealing [4]. As the present steel samples have been heat treated at high temperature $\left(1500^{\circ} \mathrm{C}\right)$ and then water quenched, the role of the simple crystal is played here by $\delta$-ferrite (b.c.c crystal, $\mathrm{a}=0.286 \mathrm{~nm}$ ). Both $\sigma$ and $\chi$ phase precipitates are obtained by annealing at $700^{\circ} \mathrm{C}$, the $\sigma$ phase appears very quickly (several minutes) while the $\chi$-phase particles are formed after longer annealing time $(200 \mathrm{~h})[4-5]$. Though precipitation of $\sigma$ and $\chi$ phases in ferrite is well documented, the crystallographic relations have rarely been studied in details. Our work consists in a Transmission Electron Microscopy (TEM) study of precipitates. The experimental information is then used to derive the interface properties leading to the observed microstructure (interface plane, orientation relationships).

\section{INTERFACE BETWEEN SIGMA-PHASE AND FERRITE}

\subsection{TEM observations}

The $\sigma$-phase precipitates form with the $\delta$-ferrite matrix characteristic lamellar aggregates. A crystallographic study shows that the lamellas tend to be parallel to the (011) $\delta$ plane (Fig. 1). Moreover two types of welldefined orientation relationships have been identified (Fig.2a,b). Both orientation sets (001) $\sigma$ parallel to (011) $\delta$. In addition, the O.Rs shown in Fig. 2 are characterized respectively by [-112] $\delta / /$ [110] $\sigma$ (Fig. 2a) and $[001] \delta / /[-110] \sigma$ (Fig. 2b).

Let us recall that the $\sigma$-phase is a complex crystal with a tetragonal cell $(a=0.88 \mathrm{~nm}, c=0.45 \mathrm{~nm})$ containing 30 atoms topologically packed (10 of them are icosahedrally coordinated) [6]. The existence of orientation relationships and the lamellar aspect are then remarkable features suggesting that simple structural relation must exist between the simple $\delta$-matrix and the $\sigma$-phase. Besides, the above $\sigma / \delta O$.Rs are reminiscent of the ones consecutive to martensitic transformation in steels, namely the Kurdjumov-Sachs and NishiyamaWasserman O.Rs.

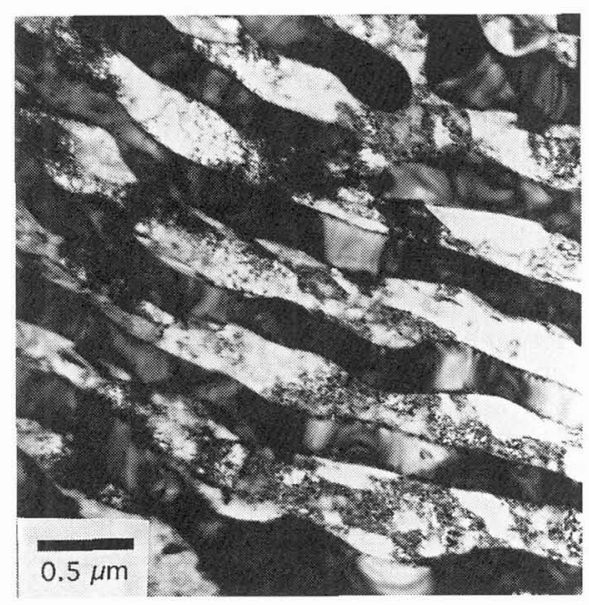

Figure 1: TEM image of the lamellar structure of the $\sigma$ fferrite aggregate. The lamellas are parallel to the $(011) \delta$ plane.

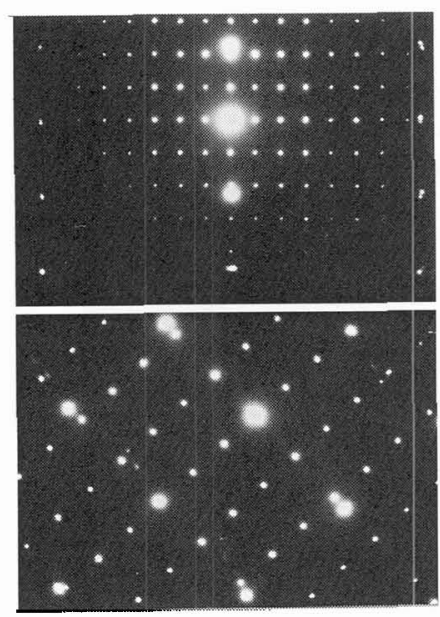

(110) $\delta / /(001) \sigma$ $[-112] \delta / /[110] \sigma$

2b)

(110) $\delta / /(001) \sigma$ $[001] \delta / /[-110] \sigma$

Figure 2: Electron diffraction patterns characteristic of the two types of $\delta / \sigma$ orientation relationships. 
When interfaces in simple crystals are studied, it is usual to consider them "edge-on". The edge-on analysis is actually not fruitful with icosahedral phase because of the absence of compact atomic planes. It is then natural to compare the structure of both phases in the interface plane. It means here comparing $(001) \sigma$ to $(011) \delta$, for instance by superimposing them (Fig. 3). Necessarily such interface analysis cannot rely on the TEM observations and must be based on the details of the atomic structure.

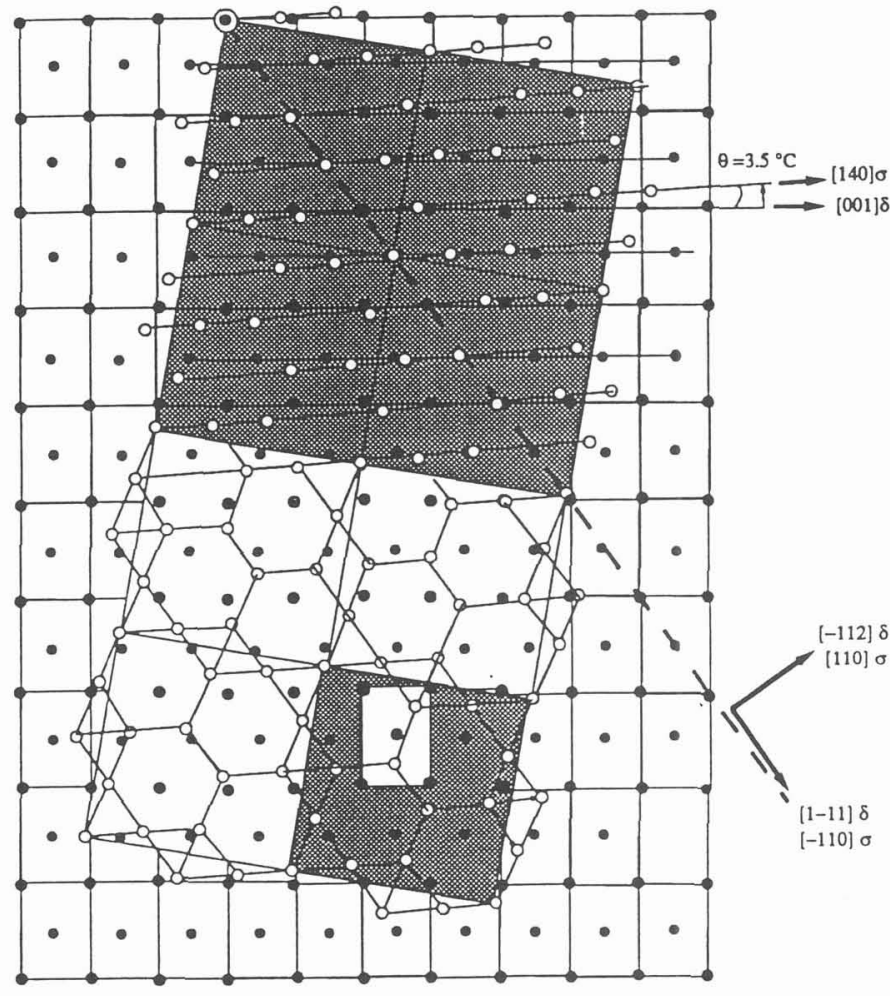

Figure 3: $(001)_{\sigma}$ atomic plane superimposed to the $(110)_{\delta}$ atomic plane according to the following orientation relationship:

$$
\begin{gathered}
(110) \delta / /(001) \sigma \\
\text { and }[-112] \delta / /[110] \sigma
\end{gathered}
$$

These planes do not have the same site density:

- in $(110) 8: 2$ atoms/cell and cell surface $\sqrt{2}\left(a_{\delta}\right)^{2}=1.16 \mathrm{~nm}^{2}$.

the (110) $\delta$ planar atomic density is 17.2 atoms.nm-2.

- in (001)o: 11 atoms/cell and cell surface $\left(a_{\sigma}\right)^{2}=7.744 \mathrm{~nm}^{2}$. the $(001) \sigma$ planar atomic density is 14.2 atoms.nm ${ }^{-2}$.

\subsection{Geometrical analysis of the interface}

At first, the two structures $((001) \sigma$ and $(011) \delta)$ superimposed in Figure 3 seem to be very close. In fact, it is not possibie to build the $(001) \sigma$ plane by a simple transformation of the $(011) \delta$ plane. A one-to-one mapping cannot be made with bounded atomic displacements when the planes to compare do not have the same planar atomic density.

Figure 3 clearly shows that the $(001) \sigma$ and $(011) \delta$ planar atomic density are not equal in the interface plane because 4 atoms per $\sigma$-phase cell are missing. The $\sigma$-phase structure provides an evident solution since the next layer contains 4 atoms per cell (Fig.4b). Let us then consider, in the $\sigma$-phase, the atomic layer limited by two planes at $z=0$ and $z=1 / 4$. This layer is a corrugated but compact layer (see its projection in Fig. 4c) which, when projected on the interface plane, has the same density as the $(011) \delta$ atomic plane.

This equality of the site density is a key idea because this conservation ensures to have a one-to-one mapping [7]. It is possible to transform the $(011) \delta$ atomic plane by a bounded transformation $(=$ local rearrangement) into a $\sigma$-phase thick layer provided that the density of sites is unchanged.

It derives from the $\sigma / \delta$ interface analysis that thick compact layer should be considered instead of atomic planes, and the interface obeys to a very simple rule: the conservation of the site density. Of course, the densities of interest are 2-dimensional ones. The atomic (110) plane in ferrite is to compare to the projection on the interface plane of the atomic sites contained within the $\sigma$-phase thick layer.

The thick layer defined above can be alternatively described as a corrugated compact layer with a thickness smaller than the atomic distances. For instance in the $\sigma$-phase, the layer to consider is $0.11 \mathrm{~nm}$ thick. 
Thinking in terms of planes in icosahedral phases has few interests because. though the structure is topologically packed, there are no dense atomic planes and most of the lattice planes have very low planar atomic density. Consequently, only thick compact layers have a physical meaning. Besides the analysis of $\sigma / f e r r i t e$ interface described above, this notion can be also used to understand the orientation relationships with the next member of the sequence: the $\chi$-phase.

a)<smiles>[O-][Si]([O-])[O-]</smiles>

b)<smiles>[Si]=C([SiH3])[SiH3]</smiles>

c)

Figure 4: 4a) $(001) \sigma$ atomic plane at $z=0$.

4b) the $\sigma$-phase atomic thick layer limited by the planes $z=0$ ) and $z=1 / 4$ seen along the (100] direction.

4 c) Projection on the $[001]$ plane, of the $\sigma$-phase atomic thick layer limited by the planes $z=0$ and $z=1 / 4$.

When projected on the interface plane, this layer has the same density of sites as the ( 110$) \delta$ plane.

\section{MICROSTRUCTURE OF KHI PHASE PRECIPITATES}

\subsection{Orientation relationships between the $\sigma$ and $\chi$ phases}

When the steel samples are annealed at $700^{\circ} \mathrm{C}$ during 365 hours, micron sized particles with a typical defect microstructure precipitate in the $\delta$-matrix (Fig. 5). These particles have been identified by TEM as $\chi$-phase particles [8]. The $\chi$-phase is an icosahedral motif crystal, isostructural to $\alpha$-Mn (b.c.c., a $=0,9 \mathrm{~nm}, 58$ atoms per cell ) [9-10]. The $\chi$-phase precipitates observed by TEM, are characterised by a lattice parameter three times the $\delta$-ferrite parameter: $a \gamma=0.86 \mathrm{~nm}$. The $\chi$ and $\sigma$ particles coexist in the $\delta$ matrix. The deduced O.R. between the three phases is $(001) \sigma / /(011) \delta / /(011) \chi$.

This O.R. is actually unexpected. The crystal structure of the $\chi$-phase (cubic, a $\chi=0.86 \mathrm{~nm}, 58$ atoms/cell) naturally suggests that the $\chi$-phase cell can be obtained by doubling the $\sigma$-phase cell (tetragonal. $a_{\sigma}=0.88 \mathrm{~nm}, c_{\sigma}=0.45 \mathrm{~nm}, 30$ atoms $/$ cell $)$. In that case, the O.R. would set $(001) \sigma$ parallel to $(001) \chi$.

Let us analyze the of $\chi$ interface in terms of planes. The atomic density in each case is computed using the following formulae: 


$$
\begin{array}{ll}
(001) \sigma \text { atomic density: } & \mathrm{n}_{\sigma} /\left(\mathrm{a}_{\sigma}\right)^{2} \\
(011) \chi \text { atomic density: } & \mathrm{n}_{\chi} / \sqrt{2}\left(\mathrm{a}_{\chi}\right)^{2}
\end{array}
$$

$a_{\chi}, n_{\chi}\left(a_{\sigma}, n_{\sigma}\right)$ are the cell parameter and number of atoms per cell in the interface plane for the $\chi$ - $(\sigma)$-phase.

The parameters being $\mathrm{a}_{\chi}=0.86 \mathrm{~nm}, \mathrm{n}_{\chi}=14$ and $\mathrm{a}_{\sigma}=0.88 \mathrm{~nm}, \mathrm{n}_{\sigma}=11$, it comes that the $(011) \chi$ and $(001) \sigma$ planes have different planar atomic densities: 13.4 atoms. $\mathrm{nm}^{-2}$ for the $(011) \chi$ plane and 14.2 atoms. $\mathrm{nm}^{-2}$ for $(001) \sigma$ plane. If we now think in terms of atomic compact layer, the previous planes have to be completed by the atoms located in their close vicinity to build a compact layer. When projected on the interface plane, this procedure will add 6 and 4 atoms per cell, respectively, for $\chi$ phase (Fig. 5) and $\sigma$-phase (Fig. 4b). The site number per unit cell in both layer, after projection on the interface plane, are now respectively $\mathrm{n}_{\chi}=20$ and $\mathrm{n}_{\sigma}=15$ leading for both layer, to the same density: 19 atoms. $\mathrm{nm}^{-2}$

As mentioned previously, the equality of densities guarantees that it is possible to transform the $(001) \sigma$ layer into the $(011) \chi$ one by a local rearrangement. The O.R. observed between the $\chi$ and $\sigma$ phases can be then understood with the same basic ideas as for the $\sigma / \delta$ interface: thick compact layer instead of atomic planes and site density conservation.

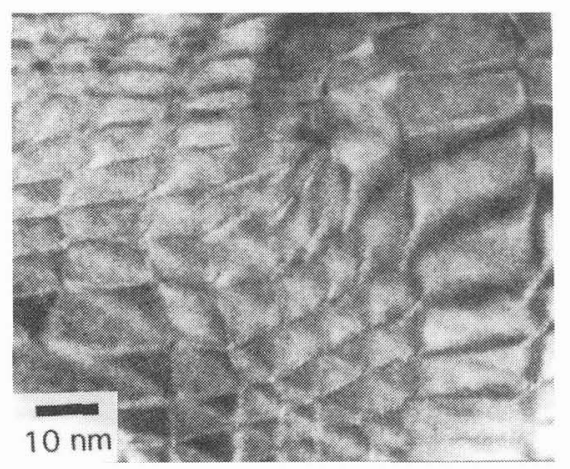

Figure 5: TEM image of a $\chi$-phase particle. Note the high density of defects forming a continuous network.

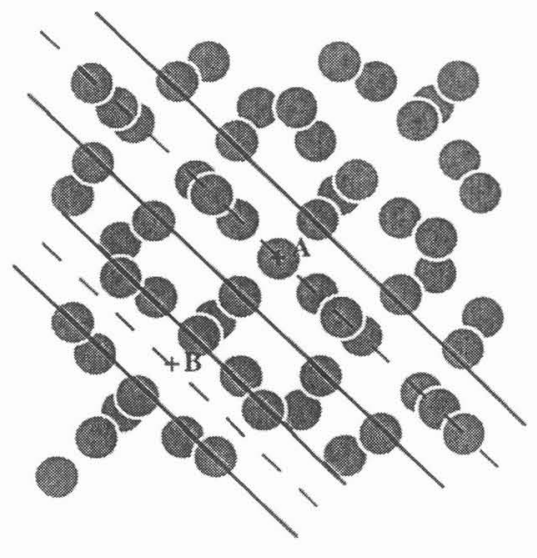

Figure 6: $\chi$-phase cell seen along the $[001]$ direction. The thick layers parallel to the (110) plane and containing either the $(1 / 2,1 / 2,1 / 2)$ node (labelled $A)$ or the $(1 / 4,1 / 4,1 / 4)$ node (labelled $B$ ) are indicated by the dotted lines.

\subsection{Antiphase boundaries of the $\chi$-phase precipitates}

The $\chi$-phase precipitates exhibit a high density of defects forming a network (Fig.5). These defects have been analyzed by TEM using the classical extinction experiments. Frequently, the defects are located in the (011) $\chi$ planes. The fault vector was identified as $\mathrm{R}=1 / 4<111>[11]$. Since the $\chi$-phase is a bcc crystal, this fault vector corresponds to a phase shift $2 \pi g$.R equal to $n \pi$ ( $g$ is a reciprocal $\chi$-phase vector). These defects are then antiphase boundaries. The $\chi$-phase structure does not suggest any simple interpretation: the $1 / 41 / 4$ $1 / 4$ nodes are not occupied. Furthermore the plane parallel to $(011) \chi$ containing the $1 / 41 / 41 / 4$ node is empty. This empty plane points out the absence of physical meaning of plane in icosahedral phases. On the contrary, if we build a thick layer containing the 1/4 1/4 1/4 node and the atoms located at less than atomic

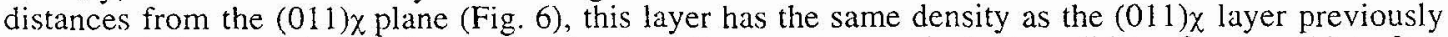
considered. It means that, in the $\chi$-phase structure, there are two equivalent candidates for a good interface with the $\sigma$ phase. Both layers are parallel to $(011) \chi$, one contains the $(1 / 2,1 / 2,1 / 2)$ node and the other the $(1 / 4,1 / 4,1 / 4)$ node. The possibility of two interfaces related by a vector $(1 / 4,1 / 4,1 / 4)$ gives then a natural interpretation for the observed antiphase boundaries. 


\section{CONCLUSION}

We report a TEM study on the precipitation of two Frank-Kasper ( $\sigma$ and $\chi$ ) phases in steels. Interface plane and orientation relationships $(\delta / \sigma, \sigma / \chi)$ have been determined. This information as well as the $\chi$-phase defects has been used to propose a simple approach for interface between simple crystal and icosahedral symmetry related crystal. Considering that lattice planes do not have a physical meaning in icosahedral phase, we emphasize on an equivalent notion: compact corrugated layer. Our analysis of interface and orientation relationships between the parent ferrite $(\delta)$ and the transformation products $(\sigma$ and $\chi)$ reveals a simple rule for the choice of the interface and O.R.: the conservation of site density in the interface plane. This conservation ensures that one layer can be transformed into the other one by local rearrangement. This approach has been developed on the example of crystalline phases with icosahedral motif decoration but the same principles hold for an interface between simple crystal and icosahedral quasicrystal.

In our experimental work, we have focussed interest on the precipitation in Fe-Cr-Mo-Ni steels. However, the observations and analysis are not limited to this system. On the contrary, they concern all transition metals intermetallic compounds because all of them exhibit the same sequence of simple and complex crystal structures. This sequence is usually understood in the frame of electronic effect (Hume-Rothery rules). The structural similarities pointed out by the present analysis give another point of view of these phases emphasizing on the geometrical aspect instead of the chemical one.

\section{References}

[1] Dahmen U., Met. and Mat. Trans. 25A (1994) 1857-1863.

[2] Frank F.C. and Kasper J.S. Acta. Cryst. 11 (1958) 184-190.

[3] Haworth C. W. and Hume-Rothery W., Phil. Mag. 3 No 33 (1958) 1013-1019.

[4] Singhall L. K. and Martin J. W., Acta. Met. 16 (1968) 1441-1451.

[5] Redjaimia A., Thesis of the University of Nancy (1991).

[6] Dickins G.J., Audrey M.B. Douglas and Taylor W.H., Acta. Cryst. 9 (1956) 297-303.

[7] Duneau, M. and Oguey, C.J. Phys. A: Math.Gen. 24 (1991) 461-475.

[8] A. Redjaïmia, J.P. Morniroli, Ultramicroscopy, 53 (1994) 305-317.

[9] Bradley A. J. and Thewlis J., Proc. Roy. Soc. A 115 (1927) 456-471.

[10] Kasper J.S., Acta. Met . 2 (1954) 456-461.

[11] Proult A. and Donnadieu P., accepted in Phil. Mag. Lett. 